\title{
A UNIVERSAL FRAMEWORK FOR PRICING FINANCIAL AND INSURANCE RISKS
}

BY

SHAUN S. WANG

\begin{abstract}
This paper presents a universal framework for pricing financial and insurance risks. Examples are given for pricing contingent payoffs, where the underlying asset or liability can be either traded or not traded. The paper also outlines an application of the framework to prescribe capital allocations within insurance companies, and to determine fair values of insurance liabilities.
\end{abstract}

\section{INTRODUCTION}

Currently there is a pressing need for a universal framework for the determination of the fair value of financial and insurance risks. In the insurance industry, this need is evident in the Society of Actuaries' "Symposium on Fair Value of Liabilities", and in the Casualty Actuarial Society's "Risk Premium Project" and "Task Force on Fair Valuing P/C Insurance Liabilities".

In the financial services industry, this pressing need is evidenced by the recent Basel Accords on regulatory risk management that require fair value, analogous to market prices, to be applied to all assets or liabilities, whether traded or not, on or off the balance sheet. In light of all these current events, this paper addresses a very timely subject.

The paper is comprised of three parts, summarized as follows:

Part One: The Framework introduces a new transform and correlation measure that extends CAPM to pricing all kinds of assets and liabilities, having any type of probability distribution, whether traded or underwritten, in finance or insurance. This transform is just as easily applied to contingent payoffs that are co-monotone with their underlying assets or liabilities.

In its simplest form, the new transform relies on a parameter called the "market price of risk", extending a familiar concept in CAPM to risks with non-normal distributions. The "market price of risk" can either be applied to,

1 SCOR, One Pierce Place, Itasca, IL 60143, E-mail: swang@scor.com 
or implied from, a distribution, in order to arrive at a "risk-adjusted price" for the underlying risk in question. The "market price of risk" increases continuously with duration, and is consistent at each horizon date between an underlying and its co-monotone contingent payoff.

When the return for an underlying asset has a normal distribution, the new transform replicates the CAPM price for that underlying asset, and recovers the Black-Scholes price for options on that underlying asset.

Part Two: Examples of Pricing Contingent Payoffs illustrates the application of the new framework to pricing call options on traded stocks, and to pricing weather derivatives.

Part Three: Capital Allocation \& Fair Values of Liabilities illustrates the application of the new framework to insurance company capital allocations, and to the determination of fair values of insurance liabilities. In particular, it addresses a challenging issue concerning the long-term duration of liabilities. Also, the framework is equally applicable to primary insurance business and excess-of-loss reinsurance when calculating fair values of liabilities.

\section{Part One. The Framework}

\section{Capital Asset Pricing Model}

CAPM is a set of predictions concerning equilibrium expected returns on assets. Classic CAPM assumes that all investors have the same one-period horizon, and asset returns have multivariate normal distributions. For a fixed time horizon, let $R_{i}$ and $R_{M}$ be the rate-of-return for asset $i$ and the market portfolio $M$, respectively. Classic CAPM asserts that

$$
E\left[R_{i}\right]=r+\beta_{i}\left\{E\left[R_{M}\right]-r\right\}
$$

where $r$ is the risk-free rate-of-return and

$$
\beta_{i}=\frac{\operatorname{Cov}\left[R_{i}, R_{M}\right]}{\sigma_{M}^{2}}
$$

is the beta of asset $i$.

Assuming that asset returns are normally distributed and the time horizon is one period (e.g., one year), a key concept in financial economics is the market price of risk:

$$
\lambda_{i}=\frac{E\left[R_{i}\right]-r}{\sigma_{i}} .
$$

In asset portfolio management, this is also called the Sharpe Ratio, after William Sharpe. 
In terms of market price of risk, CAPM can be restated as follows:

$$
\lambda_{i}=\frac{E\left[R_{i}\right]-r}{\sigma_{i}}=\frac{\operatorname{Cov}\left[R_{i}, R_{M}\right]}{\sigma_{i} \sigma_{M}} \cdot \frac{E\left[R_{M}\right]-r}{\sigma_{M}}=\rho_{i, M} \cdot \lambda_{M},
$$

where $\rho_{i, M}$ is the linear correlation coefficient between $R_{i}$ and $R_{M}$. In other words, the market price of risk for asset $i$ is directly proportional to the correlation coefficient between asset $i$ and the market portfolio $M$.

CAPM provides powerful insight regarding the risk-return relationship, where only systematic risk deserves an extra risk premium in an efficient market. However, CAPM and the concept of "market price of risk" were developed under the assumption of multivariate normal distributions for asset returns. CAPM has serious limitations when applied to insurance pricing when loss distributions are not normally distributed. In the absence of an active market for insurance liabilities, the underwriting beta by line of business has been difficult to estimate.

\section{Option Pricing Theory}

Besides CAPM, another major financial pricing paradigm is modern option pricing theory, first developed by Fischer Black and Myron Scholes (1973).

Some actuarial researchers have noted that the payoff functions of a European call option and a stop-loss reinsurance contract are similar, and have proposed an "option-pricing" approach to pricing insurance risks. Unfortunately, the Black-Scholes formula only applies to lognormal distributions of market returns, whereas actuaries work with a large array of distributional forms.

Furthermore, there are subtle differences between option pricing and actuarial pricing (see Mildenhall, 2000). One way to better appreciate the difference between "financial asset pricing" and "insurance pricing", is to recognize the difference in types of data available for pricing.

Options pricing is performed in a world of $Q$-measure (using risk-adjusted probabilities), where the available data consists of observed market prices for related financial assets. On the other hand, actuarial pricing is conducted in a world of $P$-measure (using objective probabilities), where the available data consists of projected losses, whose amounts and likelihood need to be converted to a "fair value" price (see Panjer et al, 1998).

Because of this difference, the price of an option is determined from the minimal cost of setting up a hedging portfolio, whereas the price of insurance is based on the actuarial present value of costs, plus an additional risk premium for correlation risk, parameter uncertainty and cost of capital.

\section{A Universal Pricing Method}

Consider a financial asset or liability over a time horizon $[0, T]$. Let $X=X_{T}$ denote its future value at time $t=T$, with a cumulative distribution function 
(cdf) $F(x)=\operatorname{Pr}\{X \leq x\}$. In Wang (2000), the author proposed a universal pricing method based on the following transform:

$$
F^{*}(x)=\Phi\left[\Phi^{-1}(F(x))+\lambda\right],
$$

where $\Phi$ is the standard normal cumulative distribution. The key parameter $\lambda$ is called the market price of risk, reflecting the level of systematic risk. The transform (1) is now better known as the Wang transform among financial engineers and risk managers. The Wang transform was partly inspired by the work of several prominent actuarial researchers, including Gary Venter (1991, 1998) and Robert Butsic (1999).

For a given asset $X$ with $\operatorname{cdf} F(x)$, the Wang transform will produce a "riskadjusted" cdf $F^{*}(x)$. The mean value under $F^{*}(x)$, denoted by $\mathrm{E}^{*}[X]$, will define a risk-adjusted "fair value" of $X$ at time $T$, which can be further discounted to time zero, using the risk-free interest rate.

The Wang transform is fairly easy to numerically compute. Many software packages have both $\Phi$ and $\Phi^{-1}$ as built-in functions. In Microsoft Excel, $\Phi(y)$ can be evaluated by NORMSDIST $(y)$ and $\Phi^{-1}(z)$ can be evaluated by NORM$\operatorname{SINV}(z)$.

One fortunate property of the Wang transform is that normal and lognormal distributions are preserved:

- If $F$ has a $\operatorname{Normal}\left(\mu, \sigma^{2}\right)$ distribution, $F^{*}$ is also a normal distribution with $\mu^{*}=\mu-\lambda \sigma$ and $\sigma^{*}=\sigma$.

- If $F$ has a lognormal $\left(\mu, \sigma^{2}\right)$ distribution such that $\ln (X) \sim \operatorname{Normal}\left(\mu, \sigma^{2}\right), F^{*}$ is another lognormal distribution with $\mu^{*}=\mu-\lambda \sigma$ and $\sigma^{*}=\sigma$.

Stock prices are often modeled by lognormal distributions, which implies that stock returns are modeled by normal distributions. Equivalent results can be obtained by applying the Wang transform either to the stock price distribution, or, to the stock return distribution.

Consider an asset $i$ on a one-period time horizon. Assume that the return $R_{i}$ for asset $i$ has a normal distribution with a standard deviation of $\sigma_{i}$. Applying the Wang transform to the distribution of $R_{i}$ we get a risk-adjusted rate-ofreturn:

$$
E^{*}\left[R_{i}\right]=E\left[R_{i}\right]-\lambda \sigma_{i} .
$$

In a competitive market, the risk-adjusted return for all assets should be equal to the risk-free rate, $r$. Therefore we can infer that $\lambda=\left(E\left[R_{i}\right]-r\right) / \sigma_{i}$, which is exactly the same as the market price of risk in classic CAPM. With $\lambda$ being the market price of risk for an asset, the Wang transform replicates the classic CAPM.

\section{Unified Treatment of Assets \& Liabilities}

A liability with loss variable $X$ can be viewed as a negative asset with gain $Y=$ $-X$, and vice versa. Mathematically, if a liability has a market price of risk $\lambda$, 
when treated as a negative asset, the market price of risk will be $-\lambda$. That is, the market price of risk will have the same value but opposite signs, depending upon whether a risk vehicle is treated as an asset or liability. For a liability with loss variable $X$, the Wang transform in equation (1) has an equivalent representation.

$$
S^{*}(x)=\Phi\left[\Phi^{-1}(S(x))+\lambda\right]
$$

where $S(x)=1-F(x)$.

The following operations are equivalent:

1. Applying transform (1) with $\lambda$ to the cdf $F(x)$ of a gain variable $X$,

2. Applying transform (1) with $-\lambda$ to the cdf $F(y)$ of the loss variable $Y=-X$, and

3. Applying transform (2) with $\lambda$ to $S(y)=1-F(y)$ of the loss variable $Y=-X$.

Their equivalence ensures that the same price is obtained for both sides of a risk transaction.

If a loss variable has a $\operatorname{Normal}\left(\mu, \sigma^{2}\right)$ distribution, the Wang transform (2) will produce another normal distribution with $\mu^{*}=\mu-\lambda \sigma$ and $\sigma^{*}=\sigma$. Thus, for a loss variable with a normal distribution, the Wang transform (2) recovers the traditional standard-deviation loading principle, with the parameter $\lambda$ being the constant multiplier.

\section{A New Measures of Correlation}

According to CAPM, the market price of risk $\lambda$ should reflect the correlation of an asset with the overall market portfolio. When we generalize the concept of market price of risk to assets and liabilities with non-normal distributions, the Pearson linear correlation coefficient becomes an inadequate measure of correlation. Examples can be constructed such that a deterministic relationship has a Pearson correlation coefficient close to zero. Such an example was provided in Wang (1998):

Consider the case where $X \sim \operatorname{lognormal}(0,1)$ and $Y=(X)^{\sigma}$. Despite this deterministic relationship, the linear correlation coefficient between $X$ and $Y$ approaches zero as $\sigma$ increases to infinity. That is, $\rho_{X, Y} \rightarrow 0$ as $\sigma \rightarrow \infty$.

This also implies that correlation should not be estimated by running linear regression, unless all of the variables have normal distributions.

Now we show a new way to extend the Pearson correlation coefficient to variables with non-normal distributions. For any pair of variables $\{X, Y\}$ with distributions $F_{X}$ and $F_{Y}$, we transform them into "standard normal variables":

$$
U=\Phi^{-1}\left[F_{X}(X)\right] \text {, and } V=\Phi^{-1}\left[F_{Y}(Y)\right] \text {. }
$$


We next define a new measure of correlation between $\{X, Y\}$ as the Pearson linear correlation coefficient between these transformed "standard normal variables" $\{U, V\}$ :

$$
\rho_{X, Y}^{*}=\frac{\operatorname{Cov}(U, V)}{\sigma(U) \cdot \sigma(V)}=\operatorname{Cov}(U, V) .
$$

Now, let us reconsider the case where $X \sim \operatorname{lognormal}(0,1)$ and $Y=(X)^{\sigma}$. Consistent with this deterministic relationship, this new measure of correlation between $X$ and $Y$ is always 1 . That is, $\rho_{X, Y}^{*}=1$ for all $\sigma$ values. follows:

Using this new measure of correlation we may extend classic CAPM as

$$
\lambda_{i}=\rho_{i, M}^{*} \cdot \lambda_{M}
$$

where $\lambda_{i}$ and $\lambda_{M}$ are the respective market prices of risk in the Wang transform, without assuming normality.

\section{Pricing of Contingent Payoffs}

For an underlying risk $X$ and a function $h$, we say that $Y=h(X)$ is a derivative (or contingent payoff) of $X$, since the payoff of $Y$ is a function of the outcome of $X$. If the function $h$ is monotone, we say that $Y$ is a co-monotone derivative of $X$. For example, a European call option is a co-monotone derivative of the underlying asset; in (re)insurance, an excess layer is a co-monotone derivative of the ground-up risk.

Theoretically, the underlying risk $X$ and its co-monotone derivative $Y$ should have the same market price of risk, $\lambda$, simply because they have the same correlation (as shown by using our new measure of correlation) with the market portfolio.

In pricing a contingent payoff $Y=h(X)$, there are two ways of applying the Wang transform.

- Method I: Apply the Wang transform to the distribution $F_{X}$ of the underlying risk $X$. Then derive a risk-adjusted distribution $F_{Y}^{*}$ from $F_{X}^{*}$ using $Y^{*}=h\left(X^{*}\right)$.

- Method II: First derive its own distribution $F_{Y}$ for $Y=h(X)$. Then apply the Wang transform to $F_{Y}$ directly, using the same $\lambda$ as in Method I.

Mathematically it can be shown that these two methods are equivalent. This important result validates using the Wang transform for risk-neutral valuations of contingent payoffs.

\section{Implied $\lambda$ and the Effect of Duration}

For a traded asset, the market price of risk $\lambda$ can be estimated from observed market data. We shall now take a closer look at the implied market price of risk and how it varies with the time horizon under consideration. 
Consider a continuous time model where asset prices are assumed to follow a geometric Brownian motion (GBM). Consider an individual stock, or a stock index, $i$. The asset price $X_{i}(t)$ satisfies the following stochastic differential equation:

$$
\frac{d X_{i}(t)}{X_{i}(t)}=\mu_{i} d t+\sigma_{i} d W_{i}
$$

where $\mathrm{d} W_{i}$ is a random variable drawn from a normal distribution with mean equal to zero and variance equal to $\mathrm{d} t$. In equation (4), $\mu_{i}$ is the expected rate of return for the asset, and $\sigma_{i}$ is the volatility of the asset return. Let $X_{i}(0)$ be the current asset price at time zero. For any future time $T$, the prospective stock price $X_{i}(T)$ as defined in equation (4) has a lognormal distribution (see Hull, 1997, p. 229):

$$
X_{i}(T) / X_{i}(0) \sim \operatorname{lognormal}\left(\mu_{i} T-0.5 \sigma_{i}^{2} T, \sigma_{i}^{2} T\right) .
$$

Next we apply the Wang transform to the distribution of $X_{i}(T)$ in (5) and we get

$$
X_{i}^{*}(T) / X_{i}(0) \sim \operatorname{lognormal}\left(\mu_{i} T-\lambda \sigma_{i} \sqrt{T}-0.5 \sigma_{i}^{2} T, \sigma_{i}^{2} T\right) .
$$

For any fixed future time $T$, a "no arbitrage" condition (or simply, the market value concept) implies that the risk-adjusted future asset price, when discounted by the risk-free rate, must equal the current market price. In this continuoustime model, the risk-free rate $r$ needs to be compounded continuously. As a result, we have an implied parameter value:

$$
\lambda=\lambda_{i}(T)=\frac{\left(\mu_{i}-r\right)}{\sigma_{i}} \sqrt{T}=\sqrt{T} \cdot \lambda_{i}(1) .
$$

The implied $\lambda$ in (6) coincides with the market price of risk of asset $i$ as defined in Hull (1997, p. 290). This implied $\lambda$ is also consistent with Robert Merton's inter-temporal, continuous-time CAPM (see Merton, 1973).

It is interesting to note that the market price of risk $\lambda$ increases as the time horizon lengthens. This makes intuitive sense since the longer the time horizon, the greater the exposure to unforeseen changes in the overall market environment. This interesting result has applications in pricing long-tailed insurance where losses are not reported or settled until many years after the policy period expires.

If the evolution of incurred loss resembles geometric Brownian motion, the parameter $\lambda$ should be proportional to the square root of the time period from policy inception to the date of loss settlement. The relationship (6) between $\lambda$ and duration $T$ is very useful in calculating fair values of insurance liabilities (including loss reserve discounting) and optimizing capital allocations within an insurance company.

Applying the Wang transform with the $\lambda$ in equation (6), asset $i$ has a riskadjusted distribution

$$
X_{i}^{*}(T) / X_{i}(0) \sim \operatorname{lognormal}\left(r T-0.5 \sigma_{i}^{2} T, \sigma_{i}^{2} T\right),
$$


where both the market price of risk $\lambda_{i}$ and the expected stock return $\mu_{i}$ have dropped out from the transformed distribution $F^{*}(x)$.

\section{Recovery of the Black-Scholes Formula}

A European call option on an underlying stock (or stock index) $i$ with a strike price $K$ and exercise date $T$ is defined by the following payoff function

$$
Y=\operatorname{Call}(K)=\left\{\begin{array}{cc}
0, & \text { when } X_{i}(T) \geq K, \\
X_{i}(T)-K, & \text { when } X_{i}(T)>K .
\end{array}\right.
$$

Being a non-decreasing function of the underlying stock price, the option payoff, Call $(K)$, is co-monotone with the terminal stock price, $X_{i}(T)$; thus it has the same market price of risk as the underlying stock $i$. Therefore, the same $\lambda$ as in equation (6) should be used to price the option Call $(K)$. In other words, the price of a European call option is the expected payoff under the transformed (risk-neutral) stock price distribution $F^{*}(x)$, where the expected stock return $\mu_{i}$ is replaced by the risk-free rate $r$. The resulting option price is exactly the same as the Black-Scholes formula.

There is an analogy between an unlimited stop-loss cover with retention $K$, and a European call-option with strike price $K$. Both are co-monotone derivatives of the underlying (liability or asset) variable. By applying the Wang transform to the stop-loss variable, we get a stop-loss premium as the expected stop-loss value under the transformed ground-up loss distribution.

Likewise, the price for a European call option can be evaluated as the expected option payoff under the transformed (risk-neutral) distribution for the underlying stock price, where the expected stock-return $\mu_{i}$ does not appear in the options pricing model. Thus the Wang transform adds a new perspective to the well-known risk-neutral valuation methodology of options (see Cox and Ross, 1976).

\section{Equilibrium and Replication Perspectives}

Recall that CAPM provides an equilibrium perspective of asset prices in light of its correlation with the market portfolio. With the equilibrium perspective, a call option is co-monotone with the underlying asset, thus have the same market price of risk. Using the same market price of risk, the Wang transform produces an option price as the expected option payoff under a transformed "risk-neutral" asset distribution where the expected rate-of-return is equal to the risk-free rate-of-return.

On the other hand, modern finance presents the Black-Scholes formula via a replication perspective. The replication approach relies on the ability to create a continuous riskless hedge. If asset prices change in small amounts, it is possible to simultaneously buy an option and sell a quantity of the underlying asset, so that the combined portfolio has no risk. Note that the 
instantaneous hedge is possible only because the option is a co-monotone derivative of the underlying asset.

Emanuel Derman (1996), who had worked closely with Fischer Black, commented that "Deep inside, Fischer seemed to rely on the equilibrium approach of the capital asset pricing model as the source for his intuition about options pricing. I believe this is the way the Black-Scholes equation was originally derived, although the first derivation of the options pricing formula in the Black-Scholes article is based on valuation by replication."

The Wang transform takes the equilibrium perspective of CAPM, and yet is able to reproduce the Black-Scholes price for options on underlying assets with lognormal distributions. The Wang transform thus formalizes an intrinsic relationship between CAPM and the Black-Scholes formula, along the lines of Fischer Black's reported insights.

\section{Adjust for Parameter Uncertainty}

The foregoing theory on the Wang transform assumes that the true underlying probability distribution is known without ambiguity, which is rarely the case in real life applications.

Consider the classic sampling theory in statistics. Assume that we have $k$ independent observations from a given population with a $\operatorname{Normal}\left(\mu, \sigma^{2}\right)$ distribution. Note that $\mu$ and $\sigma$ are not directly observable, we can at best estimate $\mu$ and $\sigma$ by the sample mean $\tilde{\mu}$ and sample standard deviation $\tilde{\sigma}$. As a result, when we make probability assessments regarding a future outcome, we effectively need to use a Student-t distribution.

The Student-t distribution with $k$ degrees-of-freedom has a density

$$
f(t ; k)=\frac{1}{\sqrt{2 \pi}} \cdot c_{k} \cdot\left[1+\frac{t^{2}}{k}\right]^{-(0.5 k+1)},-\infty<t<\infty,
$$

where

$$
c_{k}=\sqrt{\frac{2}{k}} \cdot \frac{\Gamma((k+1) / 2)}{\Gamma(k / 2)} .
$$

Following the sampling theory that uses a Student-t distribution in place of a normal distribution, we suggest the following technique of adjusting for parameter uncertainty:

$$
F^{*}(x)=Q\left(\Phi^{-1}(F(x))\right)
$$

where $Q$ has a Student-t distribution with $k$ degrees-of-freedom. Note that in equation (7), no restriction is imposed on the underlying distribution $F(x)$.

It may be arguable whether the adjustment in equation (7) represents a more objective view of the risk's probability distribution, or represents a form of profit loading. Regardless of how it is perceived, empirical evidences 
often suggest that market prices do often contain an adjustment for parameter uncertainty.

Let $F(x)$ be the estimated probability distribution, before adjustment for parameter uncertainty. The combination of parameter uncertainty adjustment in equation (7) and pure risk adjustment using the Wang transform in equation (1) yields the following two-factor model:

$$
F^{*}(y)=Q\left(\Phi^{-1}(F(y))+\lambda\right),
$$

where $Q$ has a Student-t distribution with $k$ degrees-of-freedom.

In a recent empirical study, Wang (2002a) reported that the two-factor model (8) provides excellent fit to the CAT-bond and corporate bond yield spreads studied by Lane (2001). Without the Student-t adjustment, the one-factor Wang transform (1) would not be able to explain the yield spreads in the Catbond and corporate bond data.

As an alternative method of adjusting for parameter uncertainty, we can modify the best-estimate cdf $F(x)$ as follows:

$$
F^{*}(x)=\Phi\left[b \cdot \Phi^{-1}(F(x))\right]
$$

where the multiplicative factor $b$ is a positive-valued function of $F(x)$. In general, the $b$-values should be no greater than 1 for both assets and liabilities, indicating that the best-estimate volatility is being amplified.

The composite of transforms (9) and (1), incorporating both systematic risk and parameter uncertainty, produces a two-factor model:

$$
F^{*}(x)=\Phi\left[b \cdot \Phi^{-1}(F(x))+\lambda\right] .
$$

Consider the special case when $b$ is a constant and $F(x)$ is a lognormal distribution. The transform (10) amplifies the volatility parameter by a factor $1 / b$, after a location shift by $\lambda$, along the lines suggested by Butsic (1999). Gary Venter, in a private communication, has also informed the author that John Major had fitted transform (10) with constant $b$ to empirically observed property CAT treaty prices.

Note that the Student-t adjustment in equation (8) can also be written in terms of the $b$-function in equation (10):

$$
F^{*}(x)=Q\left[\Phi^{-1}(F(x))+\lambda\right]=\Phi\left[b \cdot \Phi^{-1}(F(x))+\lambda\right] .
$$

With $Q$ being a Student-t distribution in (11), the implied $b$-function has a "hump" shape over the interval $0<F(x)<1$, with the highest $b$-value in the middle when $F(x)=1 / 2$, and smaller $b$-values at the tails when $F(x)$ approaches to 0 or 1 . This effectively gives higher adjustment at the extreme tails, like for deep out-of-money contingent claims, or way beyond-a-horizon-date claim settlements, where markets are illiquid, benchmark data sparse, negotiations difficult, and the cost of keeping capital reserves is high. 


\section{Extrapolation of Tail Probabilities}

Using equation (8) or (10) to adjust for parameter uncertainty does not always work in all situations. For instance, an insurance contract might offer a $\$ 100 \mathrm{M}$ limit, with no data indicating historical losses greater than $\$ 50 \mathrm{M}$, even after trending.

In such a case, tail probabilities for losses greater than $50 \mathrm{M}$ need to be extrapolated from the estimated probabilities for losses below 50M. Extreme value theory may be a useful technique for the extrapolation (see Embrechts, et al, 1997). The Wang transform can be applied to the extrapolated tail probabilities.

\section{Portfolio Management}

Portfolio management involves active selections (deletions) of the most (least) profitable business in relation to its incremental risk to the existing portfolio. We can measure the incremental risk by applying the Wang transform to the aggregate portfolio profit/loss distributions, before and after a risk is being added. The Wang transform can also be used by a portfolio manager to identify good/bad risks by comparing their respectively implied lambdas (Sharpe Ratios) with their own benchmarks for risk/return tradeoff.

\section{Final Remarks on Part One}

So far we have introduced the Wang transform as an extension to CAPM and the Black-Scholes formula. In a follow-up paper, Wang (2002b) extends Buhlmann's (1980) equilibrium-pricing model and derives the Wang transform from a set of assumptions on the behaviors of market participants.

In Part Two and Part Three of this paper we will discuss applications of the Wang transform in pricing options, weather derivatives, insurance and in capital allocation.

\section{Part Two. Examples of Pricing Contingent Payoffs}

A contingent payoff is a contractual agreement between counter-parties, whose payment trigger and amount are determined by observed outcomes of the underlying variable. A contingent payoff is a more general type of financial instrument than an option, since the underlying variable can include non-traded assets or liabilities, statistical indices, or even physical events. Most underlying variables do not follow a lognormal distribution, making the Black-Scholes formula inappropriate for benchmark pricing. In contrast, the Wang transform is applicable to any distributional form, and can be used as a universal method for pricing all kinds of contingent payoffs.

\section{Example 1. Pricing of Options}

Asset pricing is based on anticipated future price movements. Historical returns may or may not be a good indicator of future price movement. For illustration 
purposes, we assume the availability of a robust stock price projection model utilizing historical price data and other available information. Such a stock price projection may be based on a GARCH model with due considerations to mean-reversion and other economic factors. For our illustration, such a model has produced the following sample of outcomes with equal probability weights.

The underlying is a stock index with a current price of $\$ 1326.03$. Our model has produced 20 outcomes (partially based on 5-year history of quarterly returns):

$1218.71,1309.51,1287.08,1352.47,1518.84,1239.06,1415.00,1387.64$,

$1602.70,1189.37,1364.62,1505.44,1358.41,1419.09,1550.21,1355.32$, $1429.04,1359.02,1377.62,1363.84$.

The stock index return has a mean of $4.08 \%$ and a standard deviation of $8.07 \%$. Assuming that the 3-month risk-free rate is $1.5 \%$. The empirical "Sharpe Ratio" for the 3-month time-horizon is $0.32=(4.08 \%-1.5 \%) / 8.07 \%$.

We want to price a 3-month European call option on this stock with a strike price of \$1375. Apply the Wang transform to the sample stock index distribution. By using the empirical Sharpe Ratio $\lambda=0.32$ we do not recover the current stock price, since the sample distribution deviates from a lognormal distribution. We first solve for $\lambda=0.342$ by matching the current stock price of $\$ 1326.03$. Using the resulting risk-adjusted probabilities, we obtain an expected payoff of 25.35 for the 3-month European call option with strike price \$1375. After riskfree discounting, we get an option price of $\$ 24.98$.

Further explanation of the computational steps is given in Appendix 1.

Some comments:

- The market price of risk, as calculated by $(E[R]-r) / \sigma$, is precise only when the underlying asset has a normal distribution. The Wang transform, on the other hand, can iterate a precise market price of risk for underlying assets or liabilities with any type of distribution.

- With the Wang transform, we can take advantage of a good price projection model incorporating stochastic volatilities for the underlying asset.

\section{Example 2. Pricing of Weather Derivatives}

For most weather derivatives, a payoff is contingent upon the number of observed Heating-Degree-Days (HDD) for the winter months, or Cooling-DegreeDays (CDD) for the summer months, multiplied by some notional amount. The underlying variables of weather derivatives, namely HDDs and CDDs, are not traded assets by themselves. This is in contrast to equity derivatives, where the underlying stock is usually a traded asset. To price a weather derivative, an equilibrium approach is necessary.

In winter months, extreme cold weather drives up the cost for heating. The 1999 U.S. energy crisis had boosted the demand for call options on HDDs, in 
an attempt to hedge against rising heating costs. The writers of such options need to set aside capital to fund potential payouts. Option-buyers are expected to pay a risk premium to compensate for the cost of capital for the optionwriters.

We give an example of using the Wang transform to price weather derivatives. Here we use Chicago Mercantile Exchange Weather Data - Monthly Aggregate from $1 / 1 / 1979$ to $1 / 1 / 2001$.

Table 1 is the aggregate HDDs for months of December observed at the Chicago O'Hare Station. Note that there are a total of 22 observations with a mean of 1154.7 and a standard deviation of 193.4.

TABLE 1

Monthly Aggregate Data for Chicago O'Hare Station, 1979-2000

\begin{tabular}{lllllllll}
\hline \hline Date & Dec-79 & Dec-80 & Dec-81 & Dec-82 & Dec-83 & Dec-84 & Dec-85 & Dec-86 \\
HDD & 972.5 & 1147.0 & 1244.0 & 901.0 & 1573.0 & 1055.0 & 1488.0 & 1065.5 \\
Date & Dec-87 & Dec-88 & Dec-89 & Dec-90 & Dec-91 & Dec-92 & Dec-93 & Dec-94 \\
HDD & 1018.5 & 1155.0 & 1474.5 & 1129.5 & 1077.5 & 1129.5 & 1090.5 & 938.5 \\
& & & & & & \\
Date & Dec-95 & Dec-96 & Dec-97 & Dec-98 & Dec-99 & Dec-00 & & \\
$H D D$ & 1199.5 & 1156.0 & 1040.0 & 940.5 & 1090.5 & 1517.5 & & \\
\hline \hline
\end{tabular}

Assume a notional amount of $\$ 1$ for each HDD, and consider a call option on Dec-2001-HDDs with a strike price of 1350. The payoff function can be expressed mathematically as $\max (\mathrm{HDD}-1350,0)$. In order to apply the Wang transform, we first sort the annual December HDDs in an ascending order and assign objective probabilities. Here we use historical data without adjusting for on-going trends or cycles of weather conditions. In real life applications such trends and cycles need to be considered.

The key to the application of the Wang transform boils down to the selection of the lambda value. Noting that the underlying HDDs themselves are not traded assets, there is no price available for of the underlying to infer a lambda value. Nevertheless, option writers may have a benchmark "Sharpe Ratio" to target for. For illustration, we assume a benchmark Sharpe Ratio of $\lambda=$ 0.25 . In order to generate a positive risk premium, we can apply the transform in (1) with $\lambda=-0.25$. Or equivalently we can apply the transform in (2) with $\lambda=0.25$.

For the strike level of 1350 Dec-2001-HDDs, the call option has an expected payoff of 29.68 Dec-2001-HDDs, using objective probabilities. However, the "fair value" of the option is 42.70 Dec-2001-HDDs, using transformed probabilities and before any risk-free discounting.

With a lambda value of 0.25 , call options with different strike prices can be evaluated and compared (see Table 2). 
TABLE 2

Option Prices at Various Strike Levels

\begin{tabular}{lllllll}
\hline \hline Strike & $\mathbf{1 2 5 0}$ & $\mathbf{1 3 0 0}$ & $\mathbf{1 3 5 0}$ & $\mathbf{1 4 0 0}$ & $\mathbf{1 4 5 0}$ & $\mathbf{1 5 0 0}$ \\
\hline Exp. Payoff & $\$ 47.86$ & $\$ 38.77$ & $\$ 29.68$ & $\$ 20.59$ & $\$ 11.50$ & $\$ 4.11$ \\
Price & $\$ 68.21$ & $\$ 55.45$ & $\$ 42.70$ & $\$ 29.94$ & $\$ 17.18$ & $\$ 6.59$ \\
Loading & $43 \%$ & $43 \%$ & $44 \%$ & $45 \%$ & $49 \%$ & $60 \%$ \\
\hline \hline
\end{tabular}

\section{Part Three. Capital Allocation \& Fair Values of Liabilities}

Next we discuss an application of the Wang transform to insurance capital allocation and to the calculation of fair values of liabilities. Consider an insurance company writing multiple lines of business. Assume that we already know the overall economic capital for the company, or alternatively, we have derived a total required economic capital for the company based on industry benchmarks. Our goal is to allocate the cost of capital to different lines-of-business and individual contracts. Given the long-tailed nature of insurance payment patterns, insurers are required to continuously hold capital to support the reserve liabilities. One critical issue is how to appropriately reflect the duration of insurance liabilities.

There are diverse opinions on how to quantify the cost of capital for longtailed business. Some actuaries suggest that capital needs to be committed in each year in proportion to all remaining unpaid losses, without consideration of the diversification effect among development years. The other extreme of opinion is that only a one-time allocation is needed in the first year to account for the uncertainties associated with the present value of reserves. They have dramatically different implications on pricing and present a challenging issue associated with insurance capital allocation (see Venter, 2002). Most actuaries would agree that there are diversification benefits between development years; the key questions is how to quantify them. The rest of the paper is devoted to tackling this issue using the pricing framework in Part One.

\section{Available Data}

We first consider ground-up or primary business only. We shall use the following data:

- Based on historical accident-year ultimate loss ratios, we have estimates of the loss ratio volatility for each line of business, denoted by $\sigma_{\mathrm{AY}}$.

- We have estimates of the loss payment pattern for each line of business, with an average duration, denoted by $D_{\mathrm{GU}}$. Let $R(t)$ be the portion of losses remaining unpaid by time $t$. We have $D_{G U}=\int_{0}^{\infty} R(t) d t$. 


\section{Assumptions for the Evolution of Losses}

1. The best-estimate of remaining unpaid losses evolves with the passage of time as more information becomes available. During each time period (e.g. one-year), the revised estimates of loss reserve may go up or go down, with a random nature.

2. There are two opposing arguments regarding the relative uncertainty of the remaining reserve: (a) it should increase with time as more risky claims are settled later; (b) it should decrease with time as more information becomes available. Here we assume that the relative uncertainty (coefficient of variation) of remaining reserves remains constant over time. See Philbrick (1994) for further discussion of this issue.

3. Based on the above considerations, we assume a geometric Brownian motion process for the loss reserve evolution over time. Assuming that the instantaneous per annum volatility is a constant $\sigma_{1}$, we have

$$
\sigma_{A Y}^{2}=\int_{0}^{\infty} \sigma_{1}^{2} \cdot R(t) d t=\sigma_{1}^{2} \cdot D_{G U}
$$

Thus we can estimate the per-year volatility as

$$
\sigma_{1}=\frac{\sigma_{A Y}}{\sqrt{D_{G U}}}
$$

In practice, the geometric Brownian motion assumption can be relaxed to more accurately reflect the true process for loss reserve evolution. For instance, the instantaneous volatility $\sigma(\mathrm{t})$ may change with time. For property risks, $\sigma(\mathrm{t})$ may be higher for the pricing risk (when $0<t<1$ ) than the reserving risk (when $t>1$ ), as new information will emerge during the contract period regarding catastrophe activities. For casualty risks, $\sigma(t)$ may be higher for IBNR reserves than that for case reserves. For a changing $\sigma(t)$, an average per annum volatility can be calculated by

$$
\sigma_{1}=\frac{\int_{0}^{\infty} \sigma(t) R(t) d t}{\int_{0}^{\infty} R(t) d t} .
$$

Although the mentioned refinements can be incorporated in the calibration of the insurance company capital allocation, here we will restrict ourselves to the geometric Brownian motion assumption with $\sigma(\mathrm{t})=\sigma_{1}$.

\section{Risk Measure and Cost of Capital}

We define a risk-measure to approximate the cost of capital commitment, based on the following assumptions:

(a) For a given line of business, the cost of capital per-year is proportional to the underlying per-year volatility $\sigma_{1}$, which is estimated from industry data. 
(b) According to the multi-period CAPM, the market price of risk increases with the time horizon. Let parameter $\lambda_{1}$ be the per-year "market price of risk". The multi-period CAPM says that the market price of risk for time horizon $T$ is: $\lambda_{T}=\lambda_{1} \cdot \sqrt{T}$. Intuitively this makes sense. For liability insurance, the longer the duration, the higher the uncertainty, especially with respect to judicial changes and court rulings.

(c) For a given line of business, the cost of capital is proportional to the "market price of risk" for the underlying business.

(d) With risk differences by lines of business being reflected in different values of $\sigma_{1}$, we target the same Sharpe Ratio $\lambda_{1}$ per annum for all lines of business. This is because the total amount of insurance capital is used to support all insurance contracts and is legally indivisible. This is in contrast to the case for an asset portfolio where each asset is earmarked with a specific amount of capital.

For each $\$ 1$ of expected loss for a line of business with per-annum volatility $\sigma_{1}$ and average duration of $D_{\mathrm{GU}}$, the total risk measure for ground-up insurance coverage is:

$$
\lambda_{1} \cdot \sigma_{1} \cdot D_{G U}=\lambda_{1} \cdot \frac{\sigma_{A Y}}{\sqrt{D_{G U}}} \cdot D_{G U}=\lambda_{1} \cdot \sigma_{A Y} \cdot \sqrt{D_{G U}}=\lambda_{G U} \cdot \sigma_{A Y},
$$

where $\lambda_{G U}=\lambda_{1} \cdot \sqrt{D_{G U}}$.

\section{Pricing Ground-up Insurance Contracts}

For a given line of business, to calculate the insurance premium for each $\$ 1$ ground-up expected loss, we will do the following:

(1) Calculate the discount factor $\mathrm{PV}_{G U}(1)$ : Use market risk-free interest rate and the ground-up loss payment pattern.

(2) Apply risk loading to derive a pure premium:

$$
P V_{G U}(1) \cdot\left\{1+\lambda_{1} \sigma_{1} D_{G U}\right\}
$$

The factor $\lambda_{1}$ in (12) should be calibrated from "total portfolio re-balancing" based on a target return-on-equity (TROE). In other words, for the aggregate insurance portfolio, the ratio of "the total risk load plus investment return" to the total economic capital should produce a target return-on-equity. The total allocated capital over the lifetime of this $\$ 1$ liability is

$$
\lambda_{1} \sigma_{1} D_{G U}(1+r) /(T R O E-r)
$$

For year $\mathrm{j}$, the allocated capital is

$$
\lambda_{1} \sigma_{1} P_{j}(1+r) /(T R O E-r)
$$

where $P_{j}$ is the expected percentage of losses to be paid within year $j$. 
(3) Load for expenses: suppose the total expense factor is $\theta$, we can load the pure premium by a factor of $1 /(1-\theta)$.

(4) Knowing the amount of allocated capital, we can calculate the actual return-on-equity (ROE) for any given quoted premium rate.

Remark: Assume that the ground-up accident-year loss ratio follows a Brownian motion process with a total volatility $\sigma_{\mathrm{AY}}$. Formula (12) is an approximation to the resulting premium using the Wang transform with $\lambda_{G U}=\lambda_{1} \cdot \sqrt{D_{G U}}$. Thus, for ground-up business, our risk load (and capital allocation) methodology is shown to be an approximate result of the Wang transform.

\section{Pricing Excess-of-Loss Insurance Contracts}

For excess business, we need more data than for ground-up (or primary) business. In addition to the required data for ground-up business, we need the following:

- A severity curve based on industry data or theoretical loss distributions;

- Loss payment pattern for the excess cover with an average duration $D_{\mathrm{XOL}}$, which is generally longer than the ground-up payment duration.

From the perspective of a top-down approach, this involves an allocation of overall risk load to various layers. We apply the Wang transform, with adjustment for parameter uncertainty, to the severity curve to derive risk load relativity by layer. (An example of calculating relative risk loading by layer can be found in Wang, 2000). If we fix our base layer as $(0,1 \mathrm{M}]$, we can calculate a relativity factor for any layer $(\mathrm{a}, \mathrm{b}]$ as follows:

$$
\text { layer_relativity }=\frac{\text { relative_loading_for_layer }(a, b]}{\text { relative_loading_for_base_layer }}
$$

In comparison with pricing primary insurance, we price excess-of-loss layers differently as follows:

(1) Calculate the discount factor $\mathrm{PV}_{\mathrm{XOL}}(1)$ using excess-layer loss payment pattern.

(2) Apply risk loading to get a pure premium:

$$
P V_{X O L}(1) \cdot\left\{1+\lambda_{1} \cdot \sigma_{1} \cdot D_{X O L} \cdot(\text { layer_relativity })\right\} \text {. }
$$

The total allocated capital over the lifetime of this $\$ 1$ liability is

$$
\lambda_{1} \sigma_{1} D_{X O L}(\text { layer_relativity })(1+r) /(T R O E-r),
$$

For year $\mathrm{j}$, the allocated capital is

$$
\lambda_{1} \sigma_{1}\left(\text { layer_relativity) } P_{j}(1+r) /(T R O E-r),\right.
$$

where $P_{j}$ is the expected percentage of excess-layer losses to be paid in year $j$. 


\section{Remarks:}

- In most cases we suggest the use of the top-down approach, which utilizes industry data by line of business (loss volatility, severity curve, loss payment pattern). The top-down approach is based on the principle of CAPM. In other words, with the top-down approach, only non-diversifiable risks for the industry are priced into the contracts. Bault (1995) argued why industry data, rather than individual company data, should be used for pricing purposes.

- For property catastrophe (CAT) covers, modern CAT modeling techniques often use a "bottom-up" perspective. Given geographic concentration and amount of insurance data, commercial CAT models can provide us with a final loss exceedance curve for any given CAT coverage. This final loss exceedance curve already takes into account the potential frequency and severity of CAT events, as well as the correlation (concentration) of the book of business. Ideally, pricing of CAT covers should be based on such "bottom-up" information. The Wang transform, with adjustment for parameter uncertainty, can be applied directly to the loss exceedance curve for the CAT cover.

- The outlined approach is based on the pricing framework using the Wang transform. For pricing ground-up business, the Wang transform extends the classic CAPM in that the parameter $\lambda$ can now be calibrated from overall industry capital requirements. For pricing excess-of-loss layers, the Wang transform implies risk-load relativity by layer, in parallel to the Black-Scholes formula for pricing options. For both primary and excess layers, the Wang transform prescribes a method to account for the duration of liabilities.

\section{Loss Reserve Discounting}

Consider the loss reserve liability for a given line of business. The pricing approach can be equally applied to valuation of reserve liabilities. It should be kept in mind that the reserving risk, in terms of $\sigma(\mathrm{t})$, may differ from the pricing risk. Here we provide an alternative (and more direct) approach to the discount of loss reserves.

Again we assume that the loss reserve evolution follows a geometric Brownian motion. For $\$ 1$ loss reserve liability, the incurred losses at time $T$ has a distribution

$$
X(T) \sim \log \operatorname{ormal}\left(\mu T-0.5 \sigma_{1}^{2} T, \sigma_{1}^{2} T\right) .
$$

Assume that the risk-free rate is a constant $r$. The present value of the incurred losses has a distribution:

$$
X(T) \exp (-r T) \sim \log \text { ormal }\left((\mu-r) T-0.5 \sigma_{1}^{2} T, \sigma_{1}^{2} T\right) .
$$

Let $\lambda_{1}$ be the market price of risk for this line of business with one-year time horizon. For time horizon $T$, the market price of risk should be $\lambda_{1} \sqrt{T}$. Applying the Wang transform to the distribution for the discounted reserves, we get another lognormal distribution: 


$$
X^{*}(T) \exp (-r T) \sim \log \operatorname{ormal}\left(\left(\mu-r+\lambda_{1} \sigma_{1}\right) T-0.5 \sigma_{1}^{2} T, \sigma_{1}^{2} T\right) .
$$

From relation (13) we infer that applying the Wang transform is equivalent to using the following discount rate:

$$
i=r-\lambda_{1} \sigma_{1}
$$

In relation to equation (14) we make the following observations:

- The discount rate in equation (14) is the mirror formula of CAPM for assets. It is also in line with a reserve-discount formula proposed by Butsic (1988) and D'Arcy (1988).

- The per annum volatility $\sigma_{1}$ for product liability should be higher than that for worker's compensation. As a result, a lower discount rate should be used for product liabilities.

- For Worker's Compensation lifetime-pension cases, the per annum volatility $\sigma_{1}$ should be negligible and the discount rate should be close to the risk-free rate.

- With this outlined approach, the key parameter $\lambda_{1}$ can be (and should be) calibrated from aggregate industry capital allocations for each sector of underwritten business. This is in contrast to the traditional CAPM method where underwriting beta is derived from running linear regressions of equity prices of insurance firms. The "cost-of-capital" calibration of $\lambda_{1}$ should be more robust than the traditional estimation of underwriting beta.

\section{Final Comments of Part Three}

Most of the applications shown are equally applicable to banks and other financial institutions.

Our approach is mainly a top-down approach, which is consistent with CAPM. The top-down approach uses industry aggregate data, rather than relying solely on individual risk distributions. The top-down approach also eliminates any possible inconsistencies related to the treatment of frequency/ severity (see Venter, 1998).

In the CAS White Paper on Fair Valuing Property/Casualty Insurance Liabilities, several methods of estimating risk adjustments are surveyed and compared. The White Paper discussed the advantages and disadvantages of the "Distribution Transform Method", including the PH-transform method. The Wang transform can overcome most of the disadvantages listed in the White Paper:

- As shown earlier, the Wang transform can be used for producing prices or risk loads on primary business. In fact, under some common assumptions, the Wang transform reproduces the CAPM method and the Risk-Adjusted Discounting Method, which have both been used in pricing primary business.

- Unlike other transforms including the PH-transform, the Wang transform builds directly upon CAPM and Black-Scholes Theory. 
- In the Wang transform, the parameter - "market price of risk" - has been a familiar concept to financial economists. The market price of risk can be calibrated from industry capital requirements. This calibration is more robust than historical estimates of the "underwriting beta".

\section{Acknowledgments}

The author thanks two anonymous reviewers for their helpful comments and constructive suggestions. During the development of the transform in question, the author has benefited from comments by Todd Bault, Phelim Boyle, David Clark, John Kulik, John Major, Don Mango, Glenn Meyers, Stephen Mildenhall, Harry Panjer, David Ruhm, and Gary Venter. The author also thanks Julia Wirch for editorial suggestions and especially Adam Burczyk for some helpful editing. Any errors or omissions remain with the author alone.

\section{REFERENCES}

BLACK, F. and SchOles, M. (1973) The pricing of options and corporate liabilities, Journal of Political Economy, May-June, 81, 637-659.

BaulT, T. (1995) Discussion of Feldblum (1990), PCAS, LXXXII, 78-96.

BUHLMANN, H. (1980) "An economic premium principle," ASTIN Bulletin 11, 52-60.

BuTsic, R.P. (1988) Determining the proper interest rate for loss reserve discounting: an economic approach, CAS Discussion Paper Program, 147-188. It is available for download from website: http://www.casact.org/pubs/dpp/dpp88.

BuTsic, R.P. (1999) Capital Allocation for property-liability insurers: a catastrophe reinsurance application, Casualty Actuarial Society Forum, Spring 1999 Reinsurance Call Papers, 1-70.

CAS Task Force on Fair Value Liabilities, 2000, White paper on fair valuing property/casualty insurance liabilities, Casualty Actuarial Society.

Cox, J.C. and Ross, S.A. (1976) The valuation of options for alternative stochastic processes, Journal of Financial Economics 3, 145-166.

D'ARCY, S.P. (1988) Use of CAPM to discount Property-Liability loss reserves", Journal of Risk and Insurance 55:3, 481-490.

Derman, E. (1996) Reflections on Fischer, The Journal of Portfolio Management, Special Issue, December 1996. Available on the Goldman Sachs website, http://www.goldmansachs.com/ qs/doc/reflections_on_fischer.html

Embrechts, P., KLuPPelberg, C. and Mikosch, T. (1997) Modelling Extreme Events for Insurance and Finance, Springer-Verlag.

Hull, J. (1997) Options, Futures, and Other Derivatives, $3^{\text {rd }}$ edition, Prentice Hall, Upper Saddle River, New Jersey.

Merton, R.C. (1973) An intertemporal capital asset pricing model, Econometrica 41, 867 880.

Mildenhall, S. (2000) Discussion of Michael Wacek's 1997 PCAS Paper - "Application of the option market paradigm to the solution of insurance problems," Proceedings of the Casualty Actuarial Society, Volume LXXXVII. It is available on-line from http://www.casact.org/pubs/ proceed/proceed $00 /$ index.htm

Panjer, H.H. (ed.) (1998) Financial Economics, The Actuarial Foundation, Schaumburg, IL.

Philbrick, S. (1994) Accounting for risk margins, Casualty Actuarial Society Forum, Spring 1994. It will be available for download on website www.casact.org/pubs/pubs.htm.

VenTER, G.G. (1991) Premium implications of reinsurance without arbitrage, ASTIN Bulletin 21(2), 223-230. 
VENTER, G.G. (1998) Discussion of "Implementation of PH-transforms in ratemaking" by S.S. Wang, Proceedings of the Casualty Actuarial Society, Vol. LXXXV. It is available for download on website www.casact.org/pubs/pubs.htm.

Venter, G.G. (2002) Allocating Surplus - Not! CAS Actuarial Review, February 2002, 5-6.

WANG, S.S. (1998) Aggregation of correlated risks: models and algorithms, Proceedings of the Casualty Actuarial Society, Vol. LXXXV, 848-939. It is available for download on website www.casact.org/pubs/pubs.htm.

WANG, S.S. (2000) A class of distortion operators for pricing financial and insurance risks, Journal of Risk and Insurance 67(1), 15-36.

WANG, S.S. (2002a) Pricing of Catastrophe Bonds, Chapter 11 of "Alternative Risk Strategies" edited by Morton Lane, Risk Publications, London, 221-240.

WANG, S.S. (2002b) Equilibrium pricing transforms: new results of Buhlmann's 1980 economic model, presented at the 2002 AFIR Meeting in Cuncun, Mexico; to appear in ASTIN Bulletin.

\section{Appendix 1. Computational Steps for Example 1 of Part Two}

Detailed steps of the option-pricing example are shown in Table 3. We provide further explanations below:

- Column 1. Sort the sample of projected outcomes in ascending order.

- Column 2. Assign objective probabilities $f(x)=1 / 20$ to each projected outcome $\mathrm{x}$.

- Column 3. Add up the individual objective probabilities $\mathrm{f}(\mathrm{x})$ to yield a series of cumulative probabilities $\mathrm{F}(\mathrm{x})$.

- Column 4. Using the empirical Sharpe Ratio (0.32) as a "starter" lambda value, apply the Wang transform to the cumulative probabilities $\mathrm{F}(\mathrm{x})$, to yield $F^{*}(x)$.

- Column 5. De-cumulate the transformed probabilities $\mathrm{F}^{*}(\mathrm{x})$ to recover $\mathrm{f}^{*}(\mathrm{x})$. Evaluate the mean value of this projected sample using probability weights $\mathrm{f}^{*}(\mathrm{x})$. If the discounted mean value is greater (or less) than the current market value, adjust upward (or downward) the lambda value. Repeat the process of columns 4-5 until the discounted mean value matches the current market price. In this example, the "starter" lambda value of 0.320 has been tweaked to 0.342 , in order to match the current price of $\$ 1326.03$. The values of $F^{*}(x)$ and $f^{*}(x)$ shown in columns 4 and 5 are thus the final transformed probabilities using $\lambda=0.342$. Now we proceed to columns 6-8.

- Column 6. For a given strike price (\$1375 in this example), calculate the option payoff for each projected future price for the stock. That is, $y(x)=$ $\max (\mathrm{x}-1375,0)$.

- Column 7. Calculate the expected payoff by multiplying the values of the option payoff function in Column 6 by the objective probabilities in Column 2. In this example, the resulting expected payoff is $\$ 41.53$ before discounting, and $\$ 40.93$ after discounting.

- Column 8. Calculate the risk-adjusted payoff using the transformed distribution. We do that by multiplying Column (5) by Column (6). The resulting option price is $\$ 25.35$ before discounting, and $\$ 24.98$ after discounting. 
TABLE 3

Pricing of CALL-Op'TION USING the WANG TRANSForm $(\lambda=0.342)$

\begin{tabular}{|c|c|c|c|c|c|c|c|}
\hline (1) & $(2)$ & (3) & (4) & (5) & $(6)$ & (7) & (8) \\
\hline Sorted & \multicolumn{2}{|l|}{ Objective } & \multicolumn{2}{|c|}{ Transformed } & Contingent & Weighted & Risk \\
\hline Sample & \multicolumn{2}{|c|}{ Probability } & \multicolumn{2}{|c|}{ Probability } & Payoff & Value & Adjusted \\
\hline $\mathbf{x}$ & $f(x)$ & $\mathbf{F}(\mathbf{x})$ & $\mathbf{F}^{\star}(\mathbf{x})$ & $\mathbf{f}^{*}(\mathbf{x})$ & $\mathbf{y}(\mathbf{x})$ & $f(x) y(x)$ & $f^{*}(x) y(x)$ \\
\hline $1,189.37$ & 0.05000 & 0.05000 & 0.0963 & 0.0963 & - & - & - \\
\hline $1,218.71$ & 0.05000 & 0.10000 & 0.1737 & 0.0774 & - & - & - \\
\hline $1,239.06$ & 0.05000 & 0.15000 & 0.2437 & 0.0700 & - & - & - \\
\hline $1,287.08$ & 0.05000 & 0.20000 & 0.3087 & 0.0650 & - & - & - \\
\hline $1,309.51$ & 0.05000 & 0.25000 & 0.3698 & 0.0611 & - & - & - \\
\hline $1,352.47$ & 0.05000 & 0.30000 & 0.4276 & 0.0579 & - & - & -- \\
\hline $1,355.32$ & 0.05000 & 0.35000 & 0.4827 & 0.0551 & - & - & - \\
\hline $1,358.41$ & 0.05000 & 0.40000 & 0.5353 & 0.0526 & - & - & \\
\hline $1,359.02$ & 0.05000 & 0.45000 & 0.5856 & 0.0503 & - & - & - . \\
\hline $1,363.84$ & 0.05000 & 0.50000 & 0.6338 & 0.0482 & - & - & - \\
\hline $1,364.62$ & 0.05000 & 0.55000 & 0.6800 & 0.0462 & - & - & - \\
\hline $1,377.62$ & 0.05000 & 0.60000 & 0.7242 & 0.0442 & 2.62 & 0.13 & 0.12 \\
\hline $1,387.64$ & 0.05000 & 0.65000 & 0.7665 & 0.0423 & 12.64 & 0.63 & 0.53 \\
\hline $1,415.00$ & 0.05000 & 0.70000 & 0.8069 & 0.0404 & 40.00 & 2.00 & 1.62 \\
\hline $1,419.09$ & 0.05000 & 0.75000 & 0.8453 & 0.0384 & 44.09 & 2.20 & 1.69 \\
\hline $1,429.04$ & 0.05000 & 0.80000 & 0.8817 & 0.0364 & 54.04 & 2.70 & 1.97 \\
\hline $1,505.44$ & 0.05000 & 0.85000 & 0.9160 & 0.0342 & 130.44 & 6.52 & 4.47 \\
\hline $1,518.84$ & 0.05000 & 0.90000 & 0.9478 & 0.0318 & 143.84 & 7.19 & 4.57 \\
\hline $1,550.21$ & 0.05000 & 0.95000 & 0.9765 & 0.0288 & 175.21 & 8.76 & 5.04 \\
\hline $1,602.70$ & 0.05000 & 1.00000 & 1.0000 & 0.0235 & 227.70 & 11.38 & 5.34 \\
\hline \multicolumn{8}{|l|}{ Values } \\
\hline Expected & $1,380.15$ & & & $1,346.07$ & & 41.53 & 25.35 \\
\hline Discounted & $1,359.60$ & & & $1,326.03$ & & 40.91 & 24.98 \\
\hline
\end{tabular}

